\title{
Research on the Durability of Normal Strength Concrete Corroded by Different Environmental Factors
}

\author{
Xiaoping Su', a, Jianxing Wang ${ }^{1, b}$, Li Zhang ${ }^{1, \mathrm{c}}$ \\ ${ }^{1}$ Changchun Institute of Technology, Changchun, Jilin 130012, China \\ a sxp5400@163.com, b2576379550@qq.com, ${ }^{c} 120125282 @ q q . c o m$
}

\begin{abstract}
Keywords: durability, normal strength concrete, environmental factor, mass loss rate, relative dynamic elastic modulus

Abstract: With the wide application of concrete materials, the problem of concrete property degradation caused by external environment condition has drawn the attention of civil engineers. In order to study the influence of environmental factors on the durability of normal strength concrete (NSC), three groups of tests are carried out in this paper, which are multi-salt and dry-wet cycles test, multi-salt and freezing-thawing cycles test, multi-salt freezing-thawing and dry-wet cycles test. Through comparing test results about the maximum cycle numbers, mass loss rate, and relative dynamic elastic modulus obtained from each kind of test, we can draw a conclusion that the serious degradation of NSC performance is the results of joint actions of multiple environmental factors, which should be considered in the design of concrete durability.
\end{abstract}

\section{Introduction}

The badness of environment directly determines the degree of durability of concrete structure. The durability of concrete engineering under the condition of saline soil in the west of Jilin province has a great relationship with environmental factors, such as dry-wet alternation, freezing-thawing cycle, salt corrosion, and so on. In order to study the influence of environmental factors on concrete durability, three groups of tests on the durability of normal strength concrete (NSC) are carried out in this article [1-5], multi-salt and dry-wet cycles test (abbreviated as MDC), multi-salt and freezing-thawing cycles test (abbreviated as MFC), multi-salt freezing-thawing and dry-wet cycles (abbreviated as MF-DC). Da'an city, one of the most serious regions of soil salinization in Jilin province, is taken as the research region, then according to the content and type of the main dissolved salts in saline-alkali soil of Da'an, the multi-salt solution, containing $\mathrm{NaHCO}_{3}, \mathrm{NaCl}$ and $\mathrm{Na}_{2} \mathrm{SO}_{4}$, are prepared [6]. The concentration of 3.4\% is regarded as the basic concentration of erosion solution. In order to speed up the test, another two groups of multi-salt solution are made, which the concentration is $10 \%$ and $15 \%$ respectively. Furthermore, water is taken as a comparison of each group of test results. That is, the number and concentration of each group of corrosion solution is Solution $A=0$, Solution $B=3.4 \%$, Solution $C=10 \%$, and Solution $D=15 \%$.

The content and type of main dissolved salts in saline-alkali soil of Da'an are listed in Table 1.

Table 1 Content and Type of Main Dissolved Salts in Saline-alkali Soil of Da'an

\begin{tabular}{|c|c|c|c|c|c|c|c|c|c|}
\hline \multirow{2}{*}{$\begin{array}{l}\text { Sample } \\
\text { Depth } \\
\text { (cm) }\end{array}$} & \multicolumn{7}{|c|}{ Amount of Dissolved Salts (mmol/100g) } & \multirow{2}{*}{$\begin{array}{c}\text { Total } \\
\text { Amount } \\
(\%)\end{array}$} & \multirow{2}{*}{$\begin{array}{c}\text { Natural } \\
\text { Moisture } \\
\text { Content } \\
(\%)\end{array}$} \\
\hline & $\mathrm{Na}^{+}$ & $\mathrm{K}^{+}$ & $\mathrm{Ca}^{2+}$ & $\mathrm{Mg}^{2+}$ & $\mathrm{SO}_{4}^{2-}$ & $\mathrm{Cl}^{-}$ & $\mathrm{HCO}_{3}^{-}$ & & \\
\hline 20 & 7.15 & 0.35 & 0.25 & 0.17 & 1.96 & 0.95 & 4.57 & 0.60 & 12.51 \\
\hline 30 & 6.17 & 0.41 & 0.34 & 1.06 & 1.88 & 2.55 & 3.42 & 0.55 & 19.79 \\
\hline 40 & 5.07 & 0.37 & 0.19 & 0.80 & 0.97 & 2.15 & 3.14 & 0.49 & 20.68 \\
\hline 50 & 4.19 & 0.37 & 0.15 & 0.32 & 1.47 & 1.05 & 2.99 & 0.35 & 20.55 \\
\hline 70 & 3.36 & 0.19 & 0.13 & 0.40 & 0.78 & 0.97 & 2.46 & 0.28 & 20.77 \\
\hline 100 & 2.84 & 0.17 & 0.23 & 0.75 & 0.30 & 1.65 & 2.2 & 0.29 & 18.14 \\
\hline
\end{tabular}


The maximum cycle numbers of concrete, the mass loss rate, and the relative dynamic elastic modulus in each group of test, are used to compare. Among them, one time of large cycle in multi-salt freezing-thawing and dry-wet cycles test (MF-DC) corresponds to six times of multi-salt and freezing-thawing cycles (MFC) or six times of multi-salt and dry-wet cycles (MDC).

\section{The Maximum Cycle Numbers of Normal Strength Concrete in Three Groups of Tests}

The maximum cycle numbers of normal strength concrete (NSC) in three groups of tests are listed in Table 2, which corresponds to the point of concrete damage when the mass loss rate reaches $5 \%$ or the relative dynamic elastic modulus drops to $60 \%$ of the initial value. As seen from Table 2 , the maximum cycle numbers of NSC in the MF-DC test is the fewest, concrete damages most seriously and quickly. This illustrates the effect of multiple factors, such as multiple salts, dry-wet cycles, and freezing-thawing cycles on NSC damage causes the superposition corresponding, which has stronger damage capability. However the damage result cannot be regarded as the simple accumulation of each factor action.

Table 2 Maximum Cycle Number of NSC in Each Test (Times)

\begin{tabular}{cccccccccccc}
\hline \multicolumn{3}{c}{ Solution A } & \multicolumn{3}{c}{ Solution B } & \multicolumn{3}{c}{ Solution C } & \multicolumn{3}{c}{ Solution D } \\
\hline MDC & MFC & MF-DC & MDC & MFC & MF-DC & MDC & MFC & MF-DC & MDC & MFC & MF-DC \\
\hline 140 & 160 & 7 & 80 & 120 & 6 & 60 & 80 & 5 & 20 & 40 & 3 \\
\hline
\end{tabular}

\section{Comparison of the Mass Loss Rate of NSC in Three Groups of Tests}

Under different corroding conditions, concrete in each group of multi-salt solution peels off at different degrees, the mass loss rate in each operating condition is also different. The mass loss rate of NSC at the maximum cycle number in each group of tests is listed in Fig.1

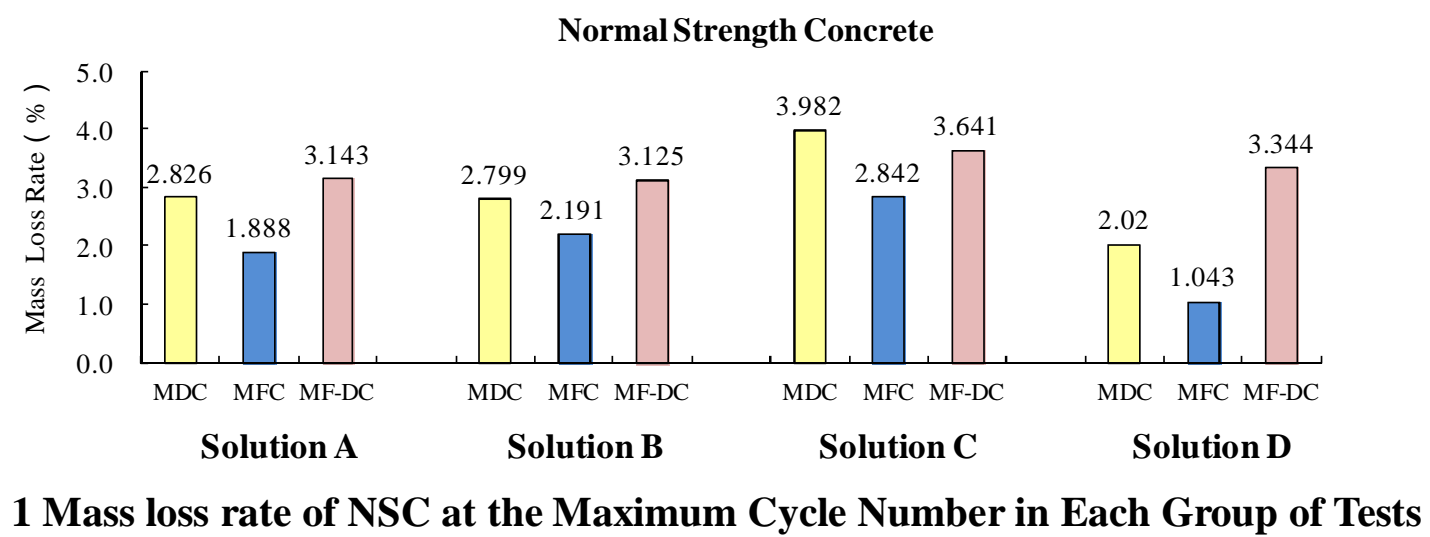

Fig. 1 Mass loss rate of NSC at the Maximum Cycle Number in Each Group of Tests

As seen from Fig. 1, the mass loss rates of NSC at the maximum cycle number in each group of tests have not reached 5\%, and the maximum value of the mass loss rate is up to $3.982 \%$. Furthermore, each group of tests has to terminate exactly because the dynamic elastic modulus of concrete drops to $60 \%$ of its initial value firstly. From the magnitude of the maximum mass loss rates of NSC, the changing law is not obvious, due to the different test condition, and the damage action and operating times imposed on NSC is so different that the loss degree of concrete mass is also different. Therefore, only from the maximum mass loss rates of NSC it is unable to distinguish the severity of concrete damage or damage law of concrete suffered from environmental factors. The mass loss rate curves of NSC in three groups of tests with the cycle times are continued to be compared, as shown in Fig. 2. 


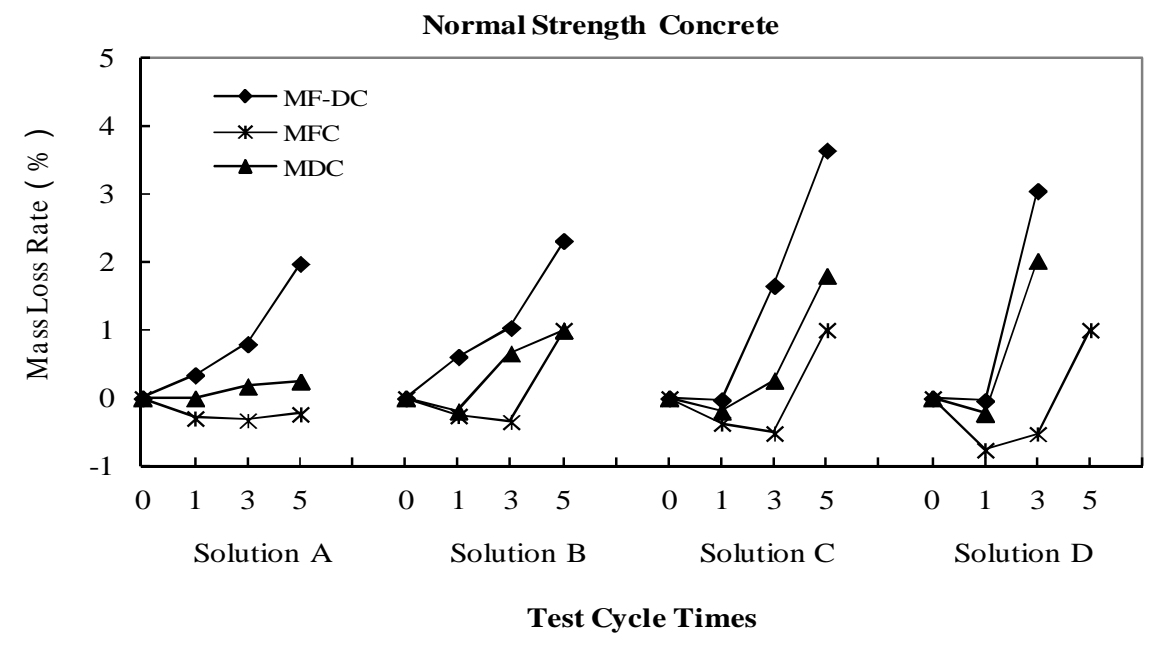

Fig. 2 Mass Loss Rate Curves of NSC in Three Groups of Tests

Seen from Fig. 2, the mass loss rate of NSC after the action of MF-DC is the most serious and fast, the second is the action of MDC and MFC. When the mass loss rate curves of NSC after the action of MDC and MFC are still at the upward stage, those mass loss rate curves of NSC after the action of MF-DC do not take on the mass increasing tendency but soon get to the mass loss stage. The greater the concentration of multi-salt solution, the steeper the mass loss rate curve, the more seriously NSC damages.

\section{Comparison of the Relative Dynamic Elastic Modulus of NSC in Three Groups of Tests}

The relative dynamic elastic modulus curves of NSC in three groups of tests are drawn in Fig.3. By contrasting, the decay laws of relative dynamic elastic modulus of NSC in each group of tests are basically same, but the curves of NSC after the action of MF-DC drop most rapidly, while the curves of NSC after MFC and MDC are still at the early increasing stage. With the increasing of the concentration of multi-salt solution, the relative dynamic elastic modulus curves of NSC drop more sharply, and concrete damage more seriously.

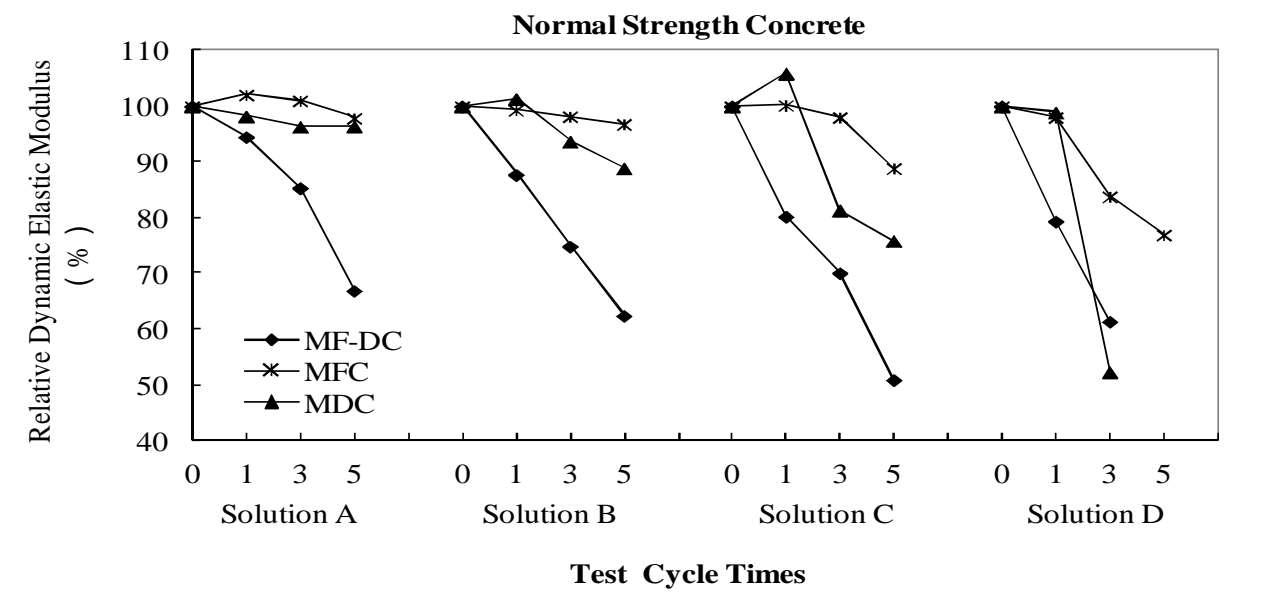

Fig. 3 Relative Dynamic Elastic Modulus Curves of NSC in Each Group of Tests

As shown in Fig.3, the changing laws of the test cycle number with solution concentration are the same. The maximum cycle times corresponding to NSC damage are gathered in Fig. 4. It is obvious that the maximum cycle times of NSC after MF-DC is much less than that of NSC after the 
action of MFC and MDC.

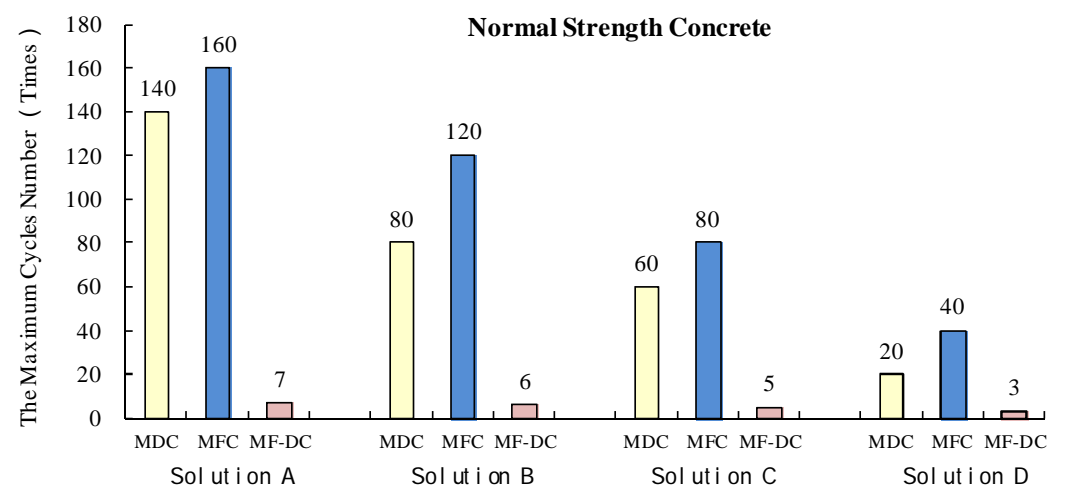

Fig. 4 Maximum Cycling Times Corresponding to NSC Damage

\section{Conclusion}

All in all, under the condition of multiple salts environment, NSC after the combined action of freezing-thawing and dry-wet cycles damage earlier and more seriously than the separate action of either freezing-thawing cycles or dry-wet cycles. Therefore, the action of various factors in service environment of concrete structures has a great influence on concrete performance. The serious degradation of NSC performance caused by the action of freezing-thawing and dry-wet cycles is the results of joint actions of multiple salts corrosion, freezing-thawing damage and of dry-wet alternation. And all sorts of action influence each other, promote each other. But concrete damage caused by multiple factors is not the simple addition of results acted by multiple salts corrosion, freezing-thawing damage and dry-wet alternation. This law has been proved by the changing laws of mass loss rate and relative dynamic elastic modulus. The durability degree of concrete is influenced by various environmental factors, the combination action of actual environmental factors should be fully considered in the design of concrete durability.

\section{Acknowledgements}

The project was financially supported by the research project of Ministry of Housing and Urban-Rural Development of China(2014-H-002), Science and Technology Department of Jilin province(20130413055GH), Jilin province Ministry of Education([2015]293), the key developing disciplines of Jilin Province, and the seed funding of Changchun Institute of Technology (320140032).

\section{References}

[1] Su Xiaoping, Wang Qing. Comparing experimental study on the concrete performance under the condition of the salt corrosion and dry-wet cycles[J]. Journal of Changchun Institute of Technology. 2012, Vol.13 (2): 1-4 (In Chinese).

[2] Su Xiaoping, Wang Qing. Experiment of the Concrete Performance Under the Condition of Multiple Salts and Dry-Wet Cycles[J]. Journal of Jilin University. 2013, Vol.43 (3): 851-857 (In Chinese).

[3] Xiaoping Su, Jinhui Guo. Study on the Durability of Normal Strength Concrete under the Action of Salt Corrosion and Dry-wet Cycles[A] The 4th International Conference on Structures and Building Materials[C] .March 15-16, 2014. Guangzhou, China. 
[4]Xiaoping Su. Analysis of Concrete Corrosion and Damage Mechanism under the Actions of Multiple Salts and Dry-Wet Cycles[A] The 4th International Conference on Civil Engineering and Building Materials[C]. May 24-25, 2014. Haikou, China.

[5] Su Xiaoping, Wang Qing, Wang Wenhua. The Frost Resistance Mechanism of Concrete under the Condition of Saline-alkali Soil in Seasonal Frozen Soil Area[J]. Journal of Jilin University. 2014, 44(4): 1244-1253( In Chinese)

[6] Zhang Jing. Changchun: Jilin University [D]. 2010 (In Chinese). 